\title{
Topological Insights into Mutant Huntingtin Exon 1 and PolyQ Aggregates by
} Cryo-Electron Tomography Jesús G. Galaz-Montoya ${ }^{1 \ddagger}$, Sarah H. Shahmoradian ${ }^{2^{\star}}$, Koning Shen ${ }^{3^{*} \dagger}$, Judith Frydman ${ }^{3}$, Wah Chiu ${ }^{1,4 \ddagger}$

6 Stanford, CA 94305, United States of America

$7 \quad{ }^{2}$ Department of Biology and Chemistry, Laboratory of Biomolecular Research, Paul

8 Scherrer Institute, Villigen, Switzerland

$9{ }^{3}$ Department of Biology, Stanford University, Stanford, CA 94305, United States of

10 America

$11{ }^{4}$ Division of CryoEM and Bioimaging, SSRL, SLAC National Accelerator Laboratory,

12 Menlo Park, CA 94025, United States of America

13

14 * Equal contribution

15 † Current address: Department of Molecular and Cell biology, University of California,

16 Berkeley, CA 94720, United States

17 ‡ Correspondence to: Jesús G. Galaz-Montoya, e-mail: jgalaz@gmail.com; Wah Chiu,

18 e-mail:wahc@stanford.edu 


\section{ABSTRACT}

Huntington disease (HD) is a neurodegenerative trinucleotide repeat disorder caused by an expanded poly-glutamine (polyQ) tract in the mutant huntingtin (mHTT) protein. The formation and topology of filamentous $\mathrm{mHTT}$ inclusions in the brain

26 (hallmarks of HD implicated in neurotoxicity) remain elusive. Using cryo-electron

27 tomography and subtomogram averaging, here we show that mHTT exon 1 and polyQonly aggregates in vitro are structurally heterogenous and filamentous, similar to prior observations with other methods. Yet, we observed some filaments in both types of

30 aggregates under $\sim 2 \mathrm{~nm}$ in width, thinner than previously reported, while other regions

31 form large sheets. In addition, our data show a prevalent subpopulation of filaments 32 exhibiting a lumpy, slab-shaped morphology in both aggregates, supportive of the "polyQ core" model. This provides a basis for future cryoET studies of various aggregated $\mathrm{mHTT}$ and polyQ constructs to improve their structure-based modeling and their identification in cells without fusion tags. 


\section{INTRODUCTION}

Huntington disease (HD) is a neurodegenerative, fatal trinucleotide repeat disorder caused by a polyQ expansion in exon 1 of mutant huntingtin (mHTT) exceeding a pathogenic threshold of $Q>\sim 35$ (MacDonald et al., 1993). Patients suffer from motor and cognitive impairments and despite our increased understanding of HD (Testa \& Jankovic, 2019) and promising clinical trials (Tabrizi et al., 2019), cures and preventive treatments remain elusive (Wild \& Tabrizi, 2017).

Expression of mHTT exon 1 (a caspase cleavage product within cells, hereafter "mEx1") elicits HD phenotypes in cellular and animal models (Mangiarini et al., 1996; von

Hörsten et al., 2003; Wang et al., 2006; Wang \& Sigworth, 2006), including primates

(Yang et al., 2008). Furthermore, mEx1 inclusions in mouse and human brains (Davies

et al., 1997; DiFiglia et al., 1997) are morphologically similar to those in R6/2 and mEx1

knock-in mice (Sathasivam et al., 2010).

A polyQ expansion in different genes causes at least eight other disorders with a similar pathogenic Q-repeat length threshold, irrespective of flanking motifs (Fan et al., 2014; Gatchel \& Zoghbi, 2005), and polyQ peptides as short as $Q=20$ are toxic when they contain a nuclear localization signal (Yang et al., 2002). Since structure often determines function (Redfern et al., 2008), as shown for mHTT toxic aggregates (Nekooki-Machida et al., 2009; Sun et al., 2015), an increased structural understanding of polyQ aggregates

63 can help uncover the mechanisms underlying their biogenesis, development, and cytotoxicity to better model polyQ disorders. 
67 various polyQ lengths (mEx1-Qn) have been amply visualized with negative staining transmission electron microscopy (NS-TEM) (Bugg et al., 2012; Crick et al., 2013; Scherzinger et al., 1999), a technique often limited to 2D projections and subject to metal stain and drying artifacts. Two recent studies used cryo focused ion beam milling and electron tomography (cryoFIB-ET) to visualize transfected mEx1-Q97 forming IBs within yeast (Gruber et al., 2018) and HeLa (Bäuerlein et al., 2017) cells. However, a green fluorescence protein (GFP) fusion tag was used, which can alter mEx1 aggregation

74 (Warner et al., 2017). cryo-electron tomography (cryoET) and subtomogram averaging (STA) (Galaz-Montoya \& Ludtke, 2017; Zhang, 2019) to visualize vitrified filamentous mEx1-Q51 and Q51 (a peptide consisting of only glutamines) aggregates in vitro. We leveraged our initial observations of mEx1-Q51 filaments by cryoET (Darrow et al., 2015; Shahmoradian et al., 2013; Shen et al., 2016) and capitalized on recent algorithmic developments including

81 compressed sensing for tomographic reconstruction (Chen et al., 2017b; Deng et al.,

82 2016), convolutional neural networks for feature annotation (Chen et al., 2017a), and

83 automated fiducial-less tiltseries alignment and subtiltseries refinement for subtomogram 84 averaging (Chen et al., 2019) to resolve previously unattainable structures. while avoiding the use of chemical fixatives and confounding fusion tags. 


\section{RESULTS}

Mutant huntingtin exon 1-Q51 filaments exhibit a large variation in width, narrow branching angles, and "lamination"

We analyzed tomographic tiltseries of vitrified mEx1-Q51 (Figure 1A), collected as previously described (see Methods)(Shahmoradian et al., 2013). Owing to the higher contrast and minimized missing wedge artifacts attainable with compressed sensing (CS) compared to standard weighted back projection (Deng et al., 2016), we incorporated CS in our pipeline to reconstruct the tiltseries into tomograms (see Methods), which exhibited aggregated filamentous densities (Figure 1B,C). While CS might introduce artifacts at very high resolution in the subnanometer range, it has been demonstrated to produce faithful reconstructions at nanometer resolution (Jin et al., 2018). The most frequently

101 observed widths from aggregates in six tomograms ranged between $\sim 5$ and $\sim 16 \mathrm{~nm}$, with

102 the thinnest filaments exhibiting regions down to $\sim 2 \mathrm{~nm}$ thickness (Figure 1D,E). On the 103 other hand, the thickest filaments measured over $\sim 20 \mathrm{~nm}$ in width. These measurements 104 are not consistent with a cylindrical shape of a single radius, as reported in recent 105 cryoFIB-ET studies (Bäuerlein et al., 2017; Gruber et al., 2018). Rather, our observations 106 are consistent with a heterogeneous plethora of thin filaments, rectangular prisms, and 107 even "sheets" of varying size. We interpret the predominant species among our observed 108 "filaments" as 3D rectangular "slabs", which could exhibit many different center-slice 109 widths in between their widest and narrowest dimensions when sliced computationally at 110 slanted angles. Our computational simulations of filamentous subtomograms using 111 EMAN2 (Galaz-Montoya et al., 2015) support this model (Supplementary Figure S1). 


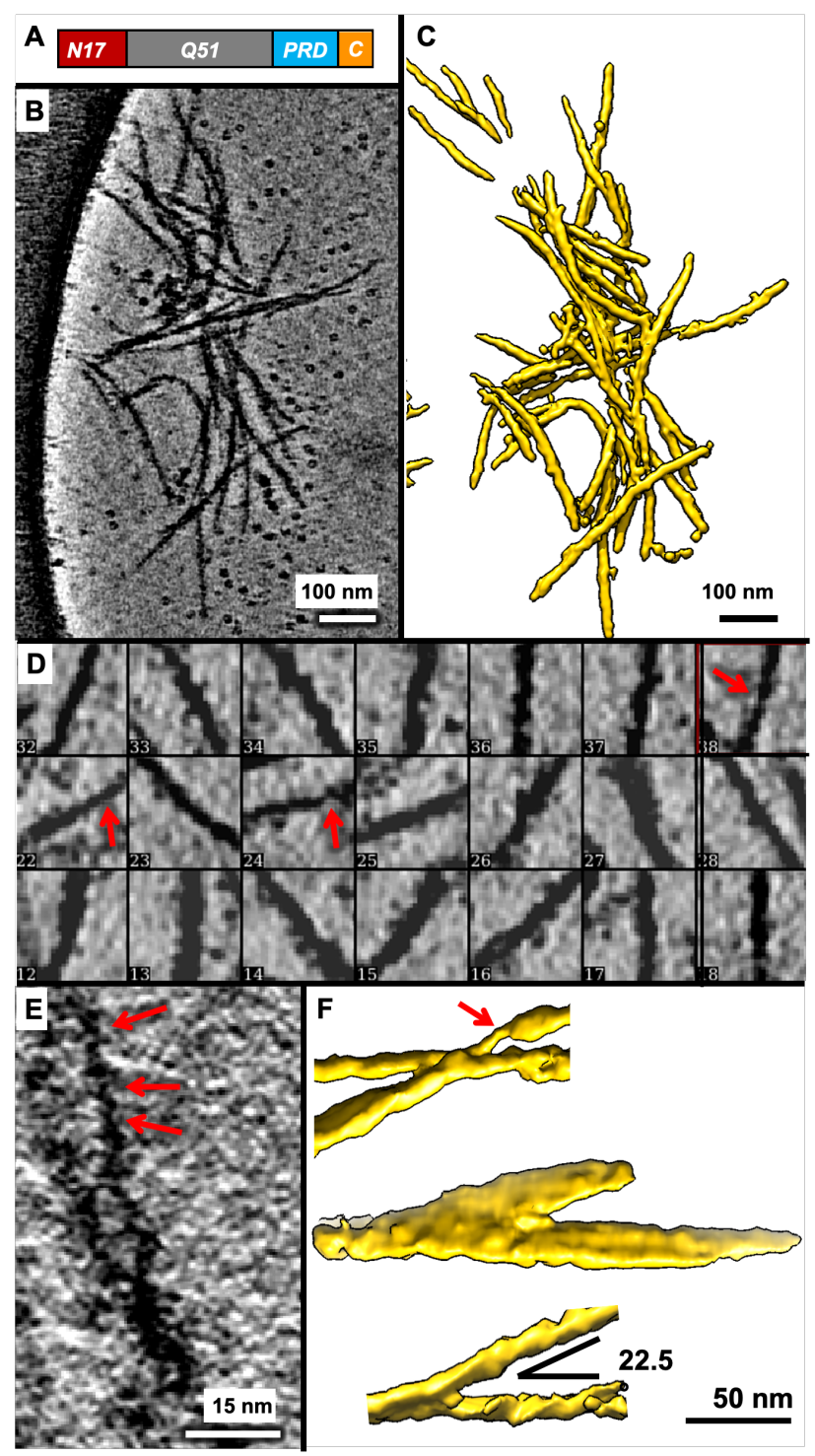

113 Figure 1. MEx1-Q51 filaments exhibit a very large variation in width within and

114 across filaments. (A) Schematic of the mEx1-Q51 construct. (B) Slice parallel to xy $(\sim 1.7$

$115 \mathrm{~nm}$ thick) through a representative $4 \mathrm{x}$ down-sampled cryoET tomogram of aggregated

116 mEx1-Q51, reconstructed with compressed sensing, lightly filtered to enhance

117 visualization, and (C) corresponding semi-automated 3D annotation. (D) Selected areas

118 from slices of large mEx1-Q51 aggregates showing individual filaments, widely varying in

119 width, with the thinnest filaments exhibiting regions down to $\sim 2 \mathrm{~nm}$ width, indicated by the 
120 red arrows. (E) Zoomed-in view of a xy slice $(\sim 0.4 \mathrm{~nm}$ thick) from a selected region of a

121 tomogram without any down-sampling, showcasing ultra-thin regions in mEx1-Q51

122 filaments. (F) Sections of annotated mEX1-Q51 filamentous aggregates from cryoET

123 tomograms showing relatively narrow branching angles and an example of a thicker

124 "laminated" sheet-like region (the annotation example in the middle).

We used semi-automated annotation based on neural networks (Chen et al.,

127 2017a) to visualize in 3D what kind of objects yielded the extensive width variations 128 detectable in 2D slices of 3D tomograms. Visualizing mEx1 filaments as 3D isosurfaces

129 (Figure 1C; Supplementary Figure S2) revealed filaments of different dimensions 130 altogether, including regions that appeared as "sheets" as thick as $\sim 50 \mathrm{~nm}$, estimated

131 from the annotations and from their persistence through $2 \mathrm{D}$ slices. The mEx1 filaments 132 seemed to predominantly branch out at angles varying from $\sim 10^{\circ}$ to $\sim 45^{\circ}$ (only 133 sporadically larger), with angles between $\sim 20^{\circ}$ to $\sim 25^{\circ}$ being most common (Figure 1F).

135 Subpopulation of mEx1-Q51 filament segments exhibits a lumpy, slab-shaped morphology Many filaments appeared to be "lumpy" both in 2D slices from 3D tomograms

138 (Figure 2A) as well as in in 3D annotations (Figure 2B), suggestive of potential 139 periodicity. Thus, we performed subtomogram averaging (STA) of filament segments, 140 avoiding obviously-laminated regions and thick bundles. The subtomogram average of 141 mEx1-Q51 filament segments ( $\mathrm{n}=450$, from 6 tomograms) converged to a lumpy $\sim 7 \times 15$ 
$142 \mathrm{~nm}$ slab at $\sim 3.5 \mathrm{~nm}$ resolution (Figure 2C). The Fourier transform of 2D re-projections of

143 the average did not reveal crisp layer lines, in agreement with previous studies suggesting

144 that mEx1 filaments do not exhibit a canonical amyloid structure with parallel subunits

145 stacked helically in register (Bugg et al., 2012). Indeed, HD does not strictly fit among the

146 diseases known as "amyloidoses" (Dobson, 2001); nonetheless, the power spectra

147 showed bright maxima off of the meridian, at $\sim 11.7 \mathrm{~nm}$ (Supplementary Figure 4A),

148 suggestive of potential periodicity for at least relatively short stretches ( $65 \mathrm{~nm}$, the length

149 included in the extracted subtomograms).
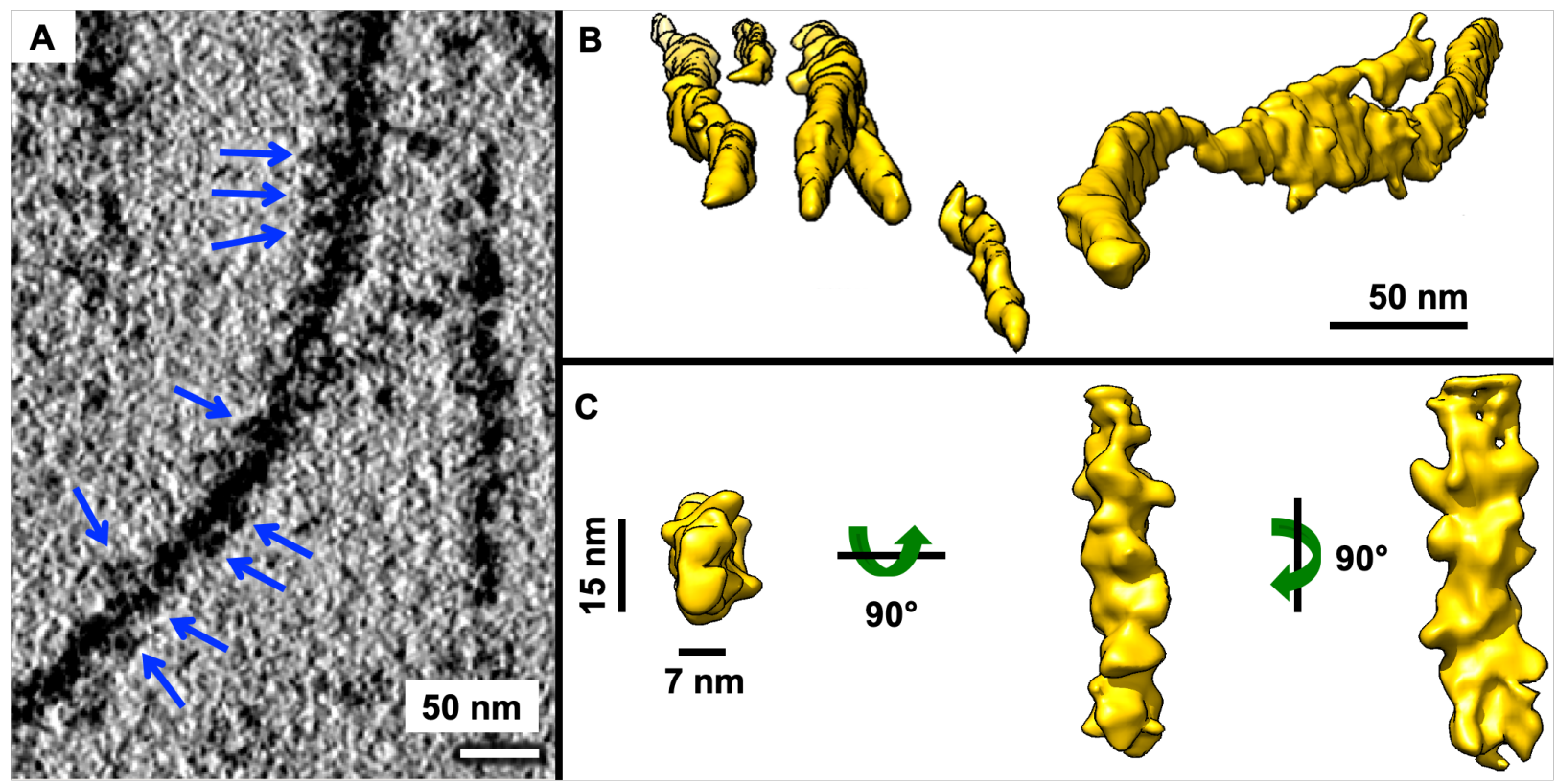

151 Figure 2. Aggregated mEx1-Q51 exhibits lumpy, slab-shaped filaments. (A) Pseudo-

152 periodic pattern of repeating "lumps" (blue arrows) along the length of an mEx1-Q51

153 filament as seen in an xy slice (4.4 A thick) from a tomogram of aggregated mEx1-Q51.

154 (B) Selected regions from semi-automated neural network annotations showing lumpy

155 filaments of various widths, including sheet-like regions (middle region of right-most 
156 example). (C) Subtomogram average of a subpopulation of filament segments exhibiting

157 a lumpy $7 \times 15 \mathrm{~nm}$ slab-shaped morphology.

159 Lumpy, slab-shaped Q51-only filaments also exhibit lamination

160 Since an expanded polyQ tract is the common culprit of all polyglutamine diseases,

161 we performed the same analyses for a Q51-only peptide (Figure 3A) as reported above

162 for mEx1-Q51. We found that Q51 also forms aggregates (Figure 3B, Supplementary

163 Figure 3) exhibiting lumpy filaments (Figure 3C) of varying widths (Figure 3D,E), with

164 regions as thin as $\sim 2 \mathrm{~nm}$.

165

166 


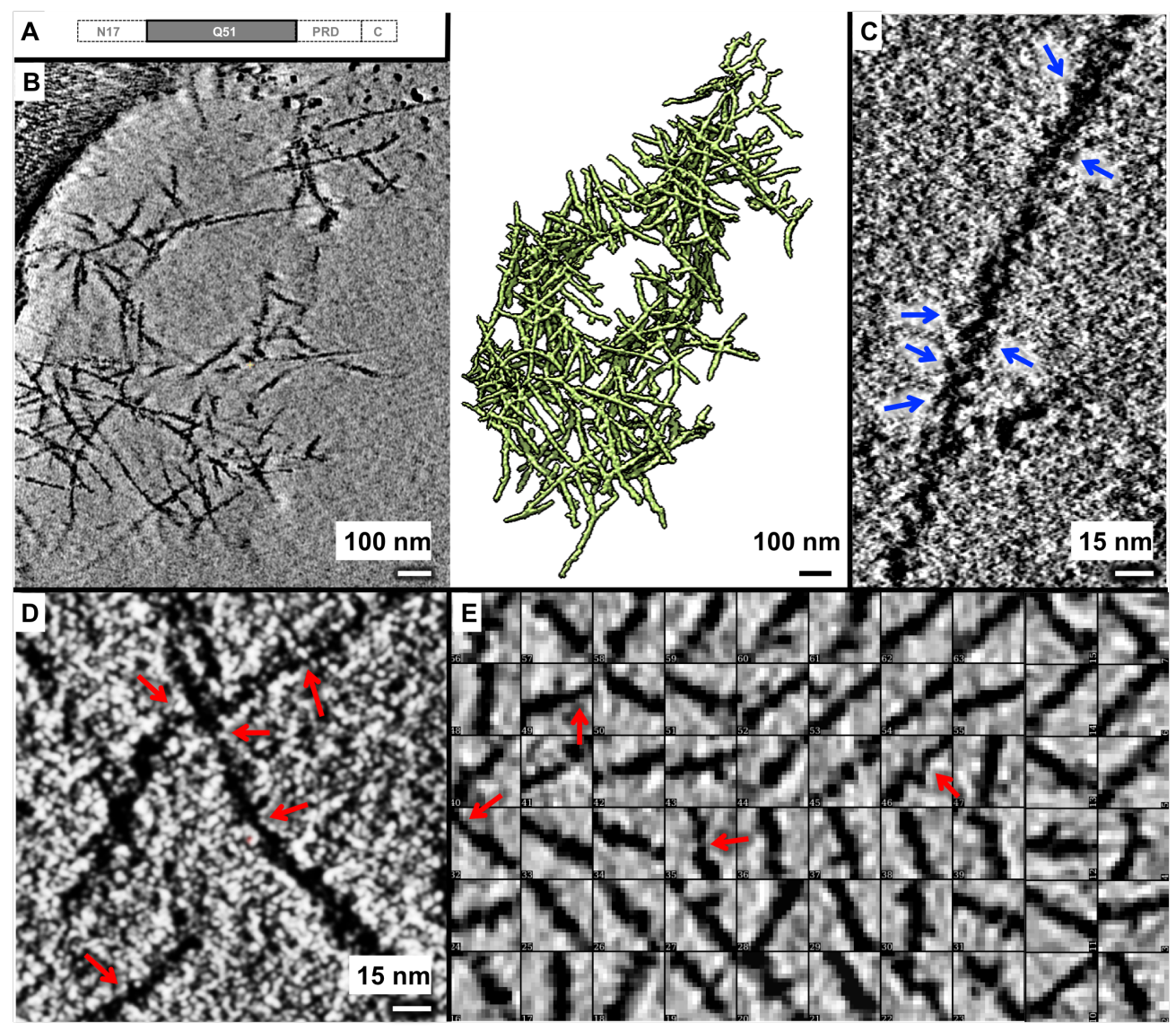

168 Figure 3. Lumpy Q51 filaments exhibit a large range of widths. (A) Schematic of the

169 Q51 construct, lacking all mEx1 domains except for the polyQ tract. (B) Slice parallel to

170 the xy plane ( 2.1 $\mathrm{nm}$ thick) through a representative $4 \mathrm{x}$ down-sampled cryoET tomogram

171 of aggregated Q51 reconstructed with compressed sensing (left) and corresponding 3D

172 annotation (right). Zoomed-in views of xy slices ( $\sim .5 \mathrm{~nm}$ thick) from selected regions of

173 the tomogram shown in A but without any down-sampling, exhibiting (C) a pseudo-

174 periodic pattern of repeating "lumps" along the length of a Q51 filament (blue arrows),

175 and (D) regions in thin filaments that are as thin as $\sim 2 \mathrm{~nm}$ in width (red arrows). (E)

176 Examples of 2D xy slices through representative 3D subtomograms of Q51 filaments

177 showing a wide variation in width, including super-thin regions $\sim 2 \mathrm{~nm}$ in width (red

178 arrows). 

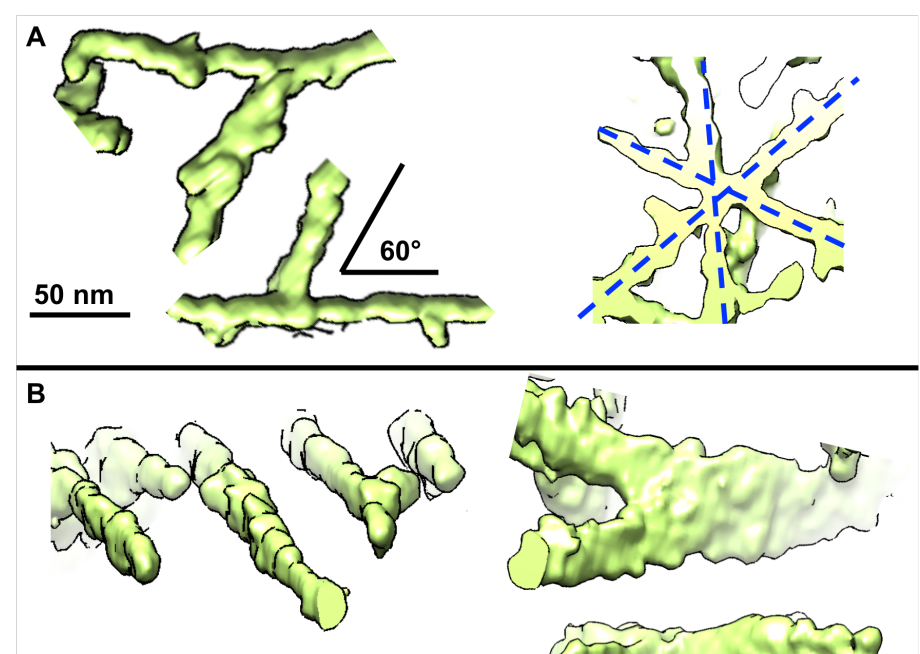

$50 \mathrm{~nm}$
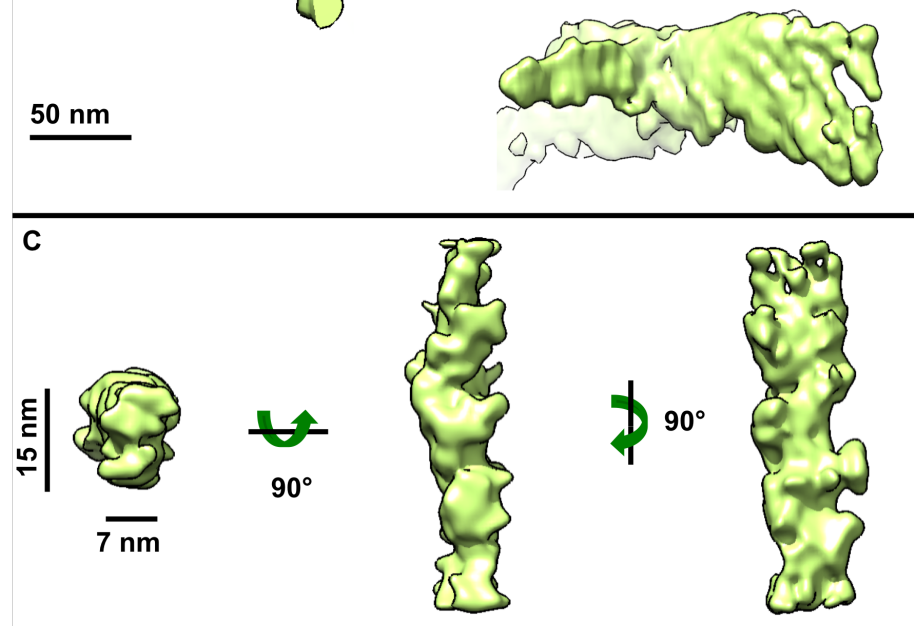

180 Figure 4. Aggregated Q51 exhibits lamination sheets and predominantly lumpy,

181 slab-shaped filaments. (A) Representative sections of annotated Q51 filamentous

182 aggregates from cryoET tomograms showing their most common branching/crossover 183 angle $\left(\sim 60^{\circ}\right)$, often in an asterisk-like pattern, and (B) thicker regions (right) akin to 184 lamination, onside thinner ones (left). (C) Subtomogram average of a subpopulation of 185 filament segments exhibiting a lumpy $7 \times 15 \mathrm{~nm}$ slab-shaped morphology. 
Q51 filaments branched out/crossed over more often and at wider angles than

mEx1-Q51 filaments, with $\sim 60^{\circ}$ being the most common angle (Figure 4A). Furthermore,

189 Q51 filaments exhibited larger lamination sheets than those of mEx1-Q51 of up to $~ 60+$

$190 \mathrm{~nm}$ in width (Figure 4B). The subtomogram average of non-laminated filament segments

191 ( $n=493$, from 6 tomograms) converged to $\sim 3.2 \mathrm{~nm}$ resolution and also revealed a lumpy

$192 \sim 7 \times 15 \mathrm{~nm}$ slab (Figure 4C), with a crossover length of $\sim 11.2 \mathrm{~nm}$ according to the power

193 spectrum of its projections (Supplementary Figure 4B), all strikingly similar results to

194 those obtained for mEx1-Q51.

\section{DISCUSSION}

In one of the earliest reports visualizing mEx1-Q51 filamentous aggregates by NS-

198 TEM, filaments digested with factor Xa or trypsin, which removes mEx1's N17 domain

199 critical to mHTT localization and function (Maiuri et al., 2013), were reported to have a 200 diameter of $\sim 7.7-12 \mathrm{~nm}$ from 2-dimensional (2D) images (Scherzinger et al., 1997). These

201 were occasionally referred to as "ribbons", and other 2D NS-TEM observations have 202 reported similar filaments with a 10-12 nm "diameter", which may associate laterally

203 (Bugg et al., 2012). However, apparent lateral associations in 2D NS-TEM observations 204 may arise from the compression of all densities into a single layer due to dehydration. In 205 contrast, the vitrified filaments seen in our 3-dimensional (3D) tomograms varied much 206 more in width within and across filaments, similar to those reported in studies visualizing 207 aggregated Q-only peptides with NS-TEM (Chen et al., 2002; Chen et al., 2001), which 208 also detected wide ribbons and thin filaments under different incubation temperatures and 209 using a "freeze-concentration" method involving cycles of freezing and thawing. Here, our 
210 observations did not suffer from NS-TEM artifacts and were conducted at $30^{\circ} \mathrm{C}$, without 211 special temperature manipulations.

While other amyloidogenic filaments have been amply studied using cryo electron 213 microscopy (cryoEM) (Fitzpatrick \& Saibil, 2019; Fitzpatrick et al., 2017; Gremer et al., 214 2017; Guerrero-Ferreira et al., 2018; ladanza et al., 2018; Li et al., 2018a; Li et al., 2018b;

215 Scheres et al., 2020), cryoEM studies of mHTT and polyQ-containing aggregates have 216 been extremely scant in comparison, likely due to the extensive conformational 217 heterogeneity of these specimens (Wetzel, 2012), which limits the applicability of "bulk 218 techniques" (e.g., Circular Dichroism) and calls for the increasing application of "single 219 molecule" techniques (Ruggeri et al., 2016). Single molecule techniques such as Atomic 220 Force Microscopy (AFM) and various modalities of electron microscopy (EM) can observe 221 individual components in aggregates (molecules, oligomers, filaments). For EM-related 222 methods, these components can be classified prior to averaging. Of note, cryoET should 223 be the method of choice for relatively thick samples exhibiting extensive conformational 224 and compositional heterogeneity as it avoids adsorption of the specimen onto 2D surfaces 225 and the confounding effect of potentially overlapping densities from different components, 226 as in 2D projections produced by single particle cryoEM.

In two recent cryoFIB-ET studies, mEx1-Q97-GFP filaments were observed and 228 annotated in transfected cellular systems but were not averaged. Rather, they were either 229 modeled as cylinders with an 8-nm diameter for template-based annotation (Bäuerlein et 230 al., 2017) or were segmented as $16 \mathrm{~nm}$ filaments (Gruber et al., 2018), surprisingly twice 231 as thick in the latter study than in the former, perhaps owing to differences in the non232 native expression systems used or to the confounding presence of GFP fusion tags. 
233 Indeed, there can be caveats to using fusions to fluorescent proteins as tags, from

234 impairing the viability and growth of cells via toxic effects from tag aggregation, excitation,

235 or photoactivation, to changing the structure, function, and cellular localization of the

236 tagged protein (Jensen, 2012). Template-based approaches have been successfully

237 applied to annotate more regularly-shaped biological filaments (Rigort et al., 2012; Rusu

238 et al., 2012); however, our data here suggest that the use of a cylindrical template is not

239 an adequate approach to annotate widely heterogeneous $\mathrm{mEx} 1$ and polyQ aggregates

240 with filamentous densities of varying dimensions. Indeed, when identifying features in

241 tomograms, template matching can be biased (Yu \& Frangakis, 2014) and manual human

242 annotation is subjective and therefore often uncertain and inconsistent (Hecksel et al.,

243 2016). On the other hand, here we used template-free, semi-automated annotation based

244 on machine learning since it can ameliorate these issues by minimizing human input and

245 the use of a priori constraints inherent in templates (Chen et al., 2017a).

246 A recent atomic force microscopy (AFM) study on mEx1-Q49 aggregation

247 suggested that nucleated branching from filaments, rather than lateral associations

248 among them, leads to large bundles (Wagner et al., 2018). However, branching does not

249 explain how the thinner (<2 $\mathrm{nm}$ thick) filaments that we observe here would assemble

250 into thicker slabs and sheets without associating laterally or growing transversally to the

251 main filament axis. Rather, our results suggest that preformed thin filaments can

252 associate laterally and/or that growing filaments can expand transversally in addition to

253 longitudinally, akin to the "lamination" observed for A $\beta$ (Lu et al., 2003), for both mEx1-

254 Q51 and Q51. 
While AFM is limited to $\sim 30 \mathrm{~nm}$ in lateral resolution of surface measurements of specimens that are often absorbed and dried onto a 2D substrate, an earlier AFM study

257 of aggregated Q44 peptide detected regions in filament tips with a "height" as thin as $\sim 5$ $258 \mathrm{~nm}$ (Poirier et al., 2002). This and the thinness of some of the filament regions we observed here (as thin as $\sim 2 \mathrm{~nm}$ ) seem to disagree with the minimum width of $\sim 7-8 \mathrm{~nm}$

260 proposed for polyQ filaments from various Qn constructs in a prior NMR study that also 261 presented NS-TEM images (Schneider et al., 2011). However, the latter study reports 262 filament widths for a Q54 peptide from NS-STEM images from 7-8 $\mathrm{nm}$ up to $\sim 16 \mathrm{~nm}$, in 263 striking agreement with the "short" and "long" sides of the slab-shaped model we propose 264 here as the predominant morphology for filaments formed by both mEx1-Q51 and Q51.

267 aggregates in cells. Furthermore, the more frequent and wider-angle branching of Q-only 268 filaments compared to mEx1 is consistent with our prior 2D cryoEM observations (Shen 269 et al., 2016), suggesting that the N17 domain promotes inter-filament bundling. 270 Conversely, the occurrence of branching might be primarily polyQ-driven. Indeed, our 3D 271 observations here, which are free from fusion tags, stains, dehydration, flattening, and 272 crystallization artifacts, provide a transforming complement and clarification to previous 273 studies by NS-TEM and AFM, as well as light microscopy (Duim et al., 2011; Duim et al., 274 2014), which visualized mEx1 filamentous aggregates at a coarser level: features often 275 described as "globules" or "oligomers" or "thick filaments" actually correspond to bundles 276 of many interwoven thinner filamentous densities when viewed by cryoET. 
The fact that the predominant populations for both mEx1 and Q-only filaments exhibit a similar lumpy slab shape and distance between putative crossovers as revealed by subtomogram averaging suggests that the morphology of their "core" is dictated by and primarily comprised of the polyQ tract, and that the flanking domains in $\mathrm{mEx} 1$ are

281 largely exposed at the filament surface, allowing them to modulate inter-filament 282 aggregation. This interpretation agrees with previous nuclear magnetic resonance (NMR) 283 studies on non-pathogenic (Sivanandam et al., 2011) and pathogenic (Hoop et al., 2014; 284 Hoop et al., 2016) mEx1 variants that propose the existence a dense polyQ core. authors observed mEx1-Q44 filaments formed at two different temperatures by 2D NS-

287 TEM images (presented in the supplement). The widths reported for these filaments were $288 \sim 6.5 \mathrm{~nm}$ and $\sim 15.2 \mathrm{~nm}$, in striking agreement with the dimensions of our slab-shaped 289 subtomogram averages of filament segments from 3D cryoET tomograms of vitrified mEX-Q51 and Q51. While their hypothesis that the thicker $\sim 15.2 \mathrm{~nm}$ filaments must arise

291 from two interwinding protofilaments $\sim 6.5 \mathrm{~nm}$ thick seems to be compatible with our 292 observations here, their model proposing that the flanking domains mediate such

293 interwinding does not explain our observation that polyQ-only filaments also yield a 294 dominant subpopulation with the same $\sim 7 \times 15 \mathrm{~nm}$ slab morphology, which could also 295 correspond to two interwoven protofilaments without flanking domains to bind them. If, 296 indeed, the mEx1-Q51 and Q51-only predominant subpopulations of $\sim 7 \times 15 \mathrm{~nm}$ filaments 297 are composed of two thinner interwinding protofilaments, our data suggest that they might 298 be bound primarily via polyQ-polyQ interactions. 
Our observations here warrant further cryoET experiments with much larger datasets of aggregation-competent mEx1 and polyQ-only constructs devoid of even

301 solubilization and purification tags, as even these can cause modest alterations in aggregation kinetics (Adegbuyiro et al., 2017; Vieweg et al., 2016). Datasets at higher magnification and contrast, using state-of-the-art instrumentation, could test whether

304 there exist filament species even thinner than the $\sim 2 \mathrm{~nm}$ regions we observed here, as 305 well as the effects of increasing polyQ length on the 3D morphology of vitrified filaments.

306 Probing the effects of post-translational modifications (PTMs) on filament and overall 307 aggregate morphology with cryoET might be of particular significance, as some PTMs 308 have been found to be modulate aggregation in vivo, with neuroprotective effects (Hegde et al., 2020). Finally, sonication concomitant with trypsin digestion of mEx1 filaments

310 might yield a homogenous-enough population of the polyQ core that may be more 311 amenable to higher-resolution cryoEM/ET studies.

\section{METHODS}

314 In vitro mEx1-Q51 and Q51 peptide aggregation assays and cryoET sample 315 preparation

We used mutant huntingtin (mHTT) exon 1 with 51 glutamine repeats (mEx1-Q51)

317 and a polyQ-only peptide with 51 repeats (Q51), each of them fused to a TEV cleavage 318 sequence and a GST tag, as previously described (Shen et al., 2016). Aggregation was

319 initiated separately at a concentration of $6 \mu \mathrm{M}$ for each construct in vitro by addition of 320 AcTEV ${ }^{\text {TM }}$ protease (Invitrogen), as previously described for mEx1-Q51 (Shahmoradian et 321 al., 2013). The samples were incubated at $30{ }^{\circ} \mathrm{C}$ before vitrification. Aliquots of $2.5 \mu \mathrm{m}$ 
322 were separately applied to 200-mesh holey carbon Quantifoil copper grids (previously

323 washed with acetone, and rinsed in PBS) between 4 and $6 \mathrm{~h}$ post-initiation of aggregation.

324 The grids were plunge-frozen in a liquid ethane bath kept at liquid nitrogen temperature

325 using a Vitrobot Mark III (FEI Instruments).

\section{Tiltseries collection}

We collected six tiltseries of the Q51 peptide using SerialEM software

328 (Mastronarde, 2005) on a JEM2100 electron microscope operated at $200 \mathrm{kV}$ from $-60^{\circ}$ to

$32960^{\circ}$ in $2^{\circ}$ increments, at $6 \mu \mathrm{m}$ target underfocus, $5.29 \AA \AA$ pixel sampling size, with a 330 cumulative dose of $\sim 80 \mathrm{e} / \AA^{2}$. We also reanalyzed a previous dataset comprised of 20

331 tiltseries of mEx1-Q51 + TRiC, collected similarly to the Q51 peptide dataset, as 332 previously described (Shahmoradian et al., 2013), with a slightly finer sampling size of $3334.4 \AA /$ pixel.

\section{Tomographic reconstruction}

All mEx1-Q51 and Q51 tilt series were binned by 2 and initially aligned and

336 reconstructed into tomograms with IMOD (Kremer et al., 1996). Images with artifacts (grid 337 bars in the field of view blocking large regions of the specimen, evident large drift, obvious

338 radiation damage, etc.) were manually removed prior to tiltseries alignment and 339 tomographic reconstruction with weighted back projection. After assessing sample 340 thickness, the tiltseries were reconstructed again into tomograms using compressed 341 sensing (CS) as implemented in ICON-GPU (Chen et al., 2017b; Deng et al., 2016) to 342 improve contrast. Of note, CS also partially restores information that is lacking due to the

343 "missing wedge" artifact inherent in all conventional single-axis limited-angle tomography 344 experiments, such as conventional cryoET (Radermacher, 1988). The tiltseries were 
345 aligned and reconstructed yet a third time for subtomogram averaging purposes (as

346 described below), using a new pipeline for cryoET in EMAN2 (Chen et al., 2019) that

347 performs "sub-tiltseries refinement", akin to prior "hybrid" methods combining concepts

348 from single particle analysis cryoEM and subtomogram averaging (Bartesaghi et al.,

349 2008; Iwasaki et al., 2005; Zhang \& Ren, 2012). We processed the mEx1-Q51 and Q51

350 datasets separately in virtually identical ways.

\section{Tomogram annotation}

Since the ultimate goal of the new EMAN2 cryoET pipeline (Chen et al., 2019) is

to perform subtiltseries refinement for subtomogram averaging, tomogram quality only

needs to be sufficient to allow for particle identification. Indeed, in EMAN2 not as many

annotations on better-quality tomograms aligned with IMOD and reconstructed with CS,

tomograms using EMAN2's neural network semi-automated annotation tools (Chen et al.,

2017a), except that $\sim 2-3 x$ as many "references" as the 10 recommended were segmented

361 to seed annotation, and $\sim 2-3 x$ as many "negative" samples as the 100 recommended

362 were selected to minimize false positives. We initially performed annotation of all mEx1-

363 Q51 and Q51 tomograms by applying the convolutional neural network from the best

364 tomogram to all the rest, separately for each specimen. However, false positives (such

365 as annotating the carbon-hole edge and/or gold fiducials) were reduced further when we

366 generated a neural network specific for each mEx1-Q51 and Q51 tomogram.

\section{Fibril width range measurements}


In all limited-angle tomography experiments (when you cannot tilt through the

entire full range from $0^{\circ}$ to $180^{\circ}$ or $-90^{\circ}$ to $+90^{\circ}$ to collect a full set of projections around

370 the object of interest), the "missing wedge" artifact worsens the resolution of raw

371 tomograms along the Z-axis compared to that in the $\mathrm{X}$ and $\mathrm{Y}$ directions, often giving the

372 appearance of "elongation" of features along the axis with lowest resolution. Therefore,

373 filament widths cannot be accurately measured in 3D from raw tomograms nor their

374 corresponding annotations in arbitrary orientations. The most conservative

375 measurements in the absence of averaging should be performed on slices along the Z-

376 axis of reconstructed tomograms (i.e., on sections parallel to the XY plane) since features

377 are much less well-resolved in the $X Z$ and $Y Z$ planes. Here, we boxed out filament

378 segments for STA (below) and manually measured the thinnest and thickest parts of

379 segments $(N$ 100) from the central $X Y$ slice of the corresponding subtomogram. The

380 mEx1-Q51 and Q51 data were separately processed in identical ways.

381 Initial model generation for subtomogram averaging (STA)

To carry out sub-tiltseries refinement, the new EMAN2 cryoET pipeline (Chen et

al., 2019) requires that all steps (from initial tomographic reconstruction) be performed in

384 EMAN2. However, as explained above, whole-tiltseries alignment with IMOD is often

385 superior in quality, given its refinement of more reconstruction parameters, and

386 reconstruction with ICON-GPU can yield higher-contrast tomograms with minimized

387 missing wedge artifacts. Therefore, to generate an initial model, we manually extracted

388 filament segments without much overlap from the best tomogram for each specimen

389 ( $n=97$ for mEx1-Q51; $n=135$ for Q51) avoiding branching points and regions of dense

390 bundling or obvious lamination. Then, we aligned these subtomograms to a cylindrical 
391 reference with a soft edge and computed the average using the legacy tools for STA in

392 EMAN2 (Galaz-Montoya et al., 2016). This average of vertically-aligned filaments was

393 then refined constraining the angular search in altitude to only allow for slightly-tilted

394 orientations (since all particles were already pre-aligned to a cylinder) and flips of $180^{\circ}$ in

395 altitude (the other two Euler angles were completely unconstrained). Alignment

396 converged in $\sim 4-5$ iterations for both datasets. We used these preliminary averages as

397 initial models for subsequent unconstrained gold-standard subtomogram averaging of

398 mEx1-Q51 and Q51 with sub-tiltseries refinement in the new EMAN2 pipeline.

\section{Subtomogram averaging}

Since the reconstruction geometry is different for tomograms produced with

401 different software packages, we had to pick subtomograms of filament segments (with <

$402 \sim 50 \%$ overlap) manually from scratch ( $n=450$ for mEx1-Q51; $n=493$ for Q51) in EMAN2-

403 reconstructed tomograms. Gold-standard refinements seeded with the initial models

404 described above converged in $\sim 4-5$ iteration and $\sim 60 \%$ of the best-correlating particles

405 were kept in the final average for each dataset. The subtiltseries refinement step alone

406 improved the resolution drastically by $\sim 10 \AA$ or more for both datasets, yielding averages

407 at $\sim 3.5 \mathrm{~nm}$ and $\sim 3.2 \mathrm{~nm}$ resolution for mEx1-Q51 and Q51, respectively, according to the 408 gold-standard FSC $=0.143$ criterion. 


\section{ACCESSION NUMBERS AND DATA ACCESSIBILITY}

The Electron Microscopy Data Bank accession numbers for the structures reported

415 in this paper are as follows: mEx1-Q51 subtomogram average, EMD-21248; Q51

416 subtomogram average, EMD-21253. The raw data can be made accessible upon request.

\section{AUTHOR CONTRIBUTIONS}

All authors planned and designed experiments. K.S. generated and purified the

$420 \mathrm{mEx} 1-\mathrm{Q} 51$ and Q51 constructs. S.H.S. collected the cryoET data. J.G.G.M. performed all

421 data processing and analyses, prepared all figures, and wrote the manuscript with

422 feedback from all authors.

423

424 ACKNOWLEDGEMENTS AND FUNDING

USA, No. NS092525 to J.F. and W.C., and No. P41GM103832 to W.C.

\section{CONFLICTS OF INTEREST DECLARATIONS}

429 The authors declare no conflicts of interest. 


\section{SUPPLEMENTARY DATA}

A
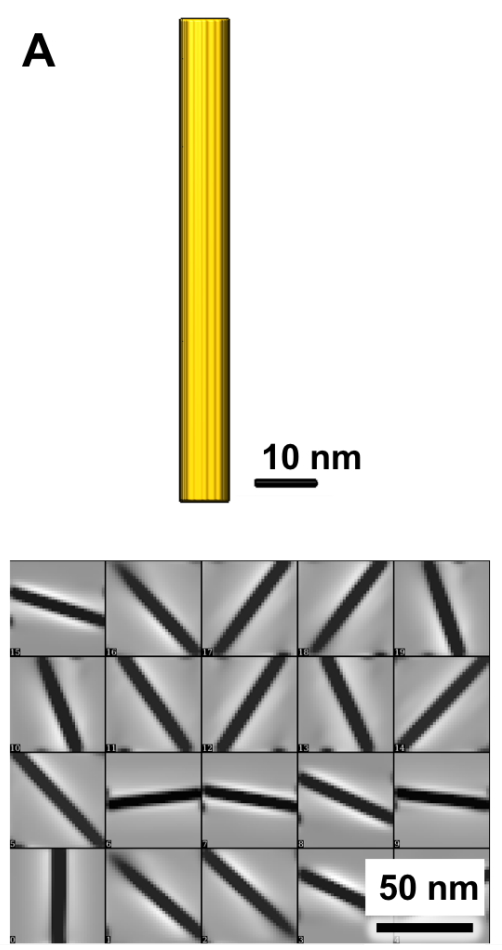
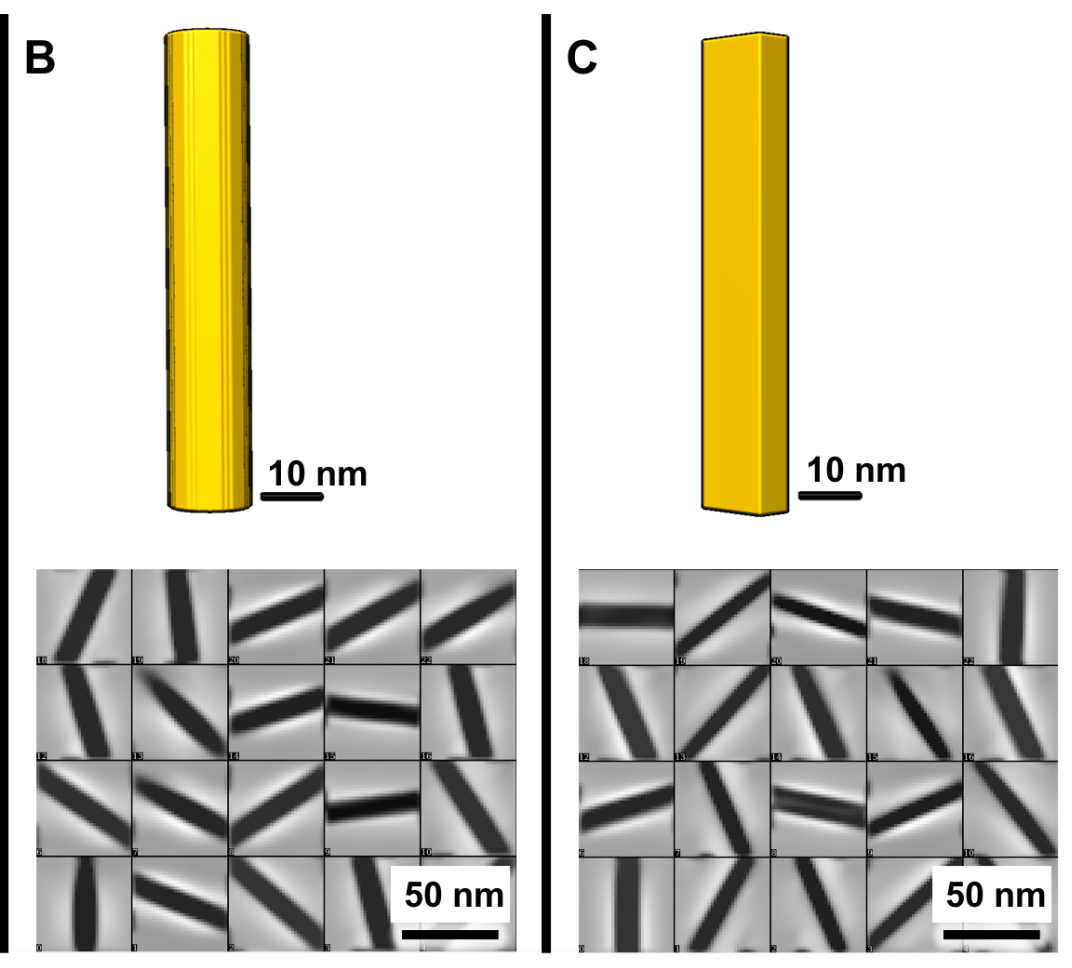

435 Supplementary Figure S1. Slab-shaped filaments are more consistent with

436 observations of variable width in central $\mathbf{Z}$ cross-sections (in the $\mathrm{XY}$ plane,

437 unaffected by the "missing wedge" artifact) than cylindrical filaments. Simulated

438 model and corresponding central $Z$ cross-sections of simulated subtomograms for a

439 cylinder (A) $7 \mathrm{~nm}$ or (B) $15 \mathrm{~nm}$ in diameter, and (C) a rectangular slab with narrow and

440 wide sides measuring 7 and $15 \mathrm{~nm}$, respectively. 

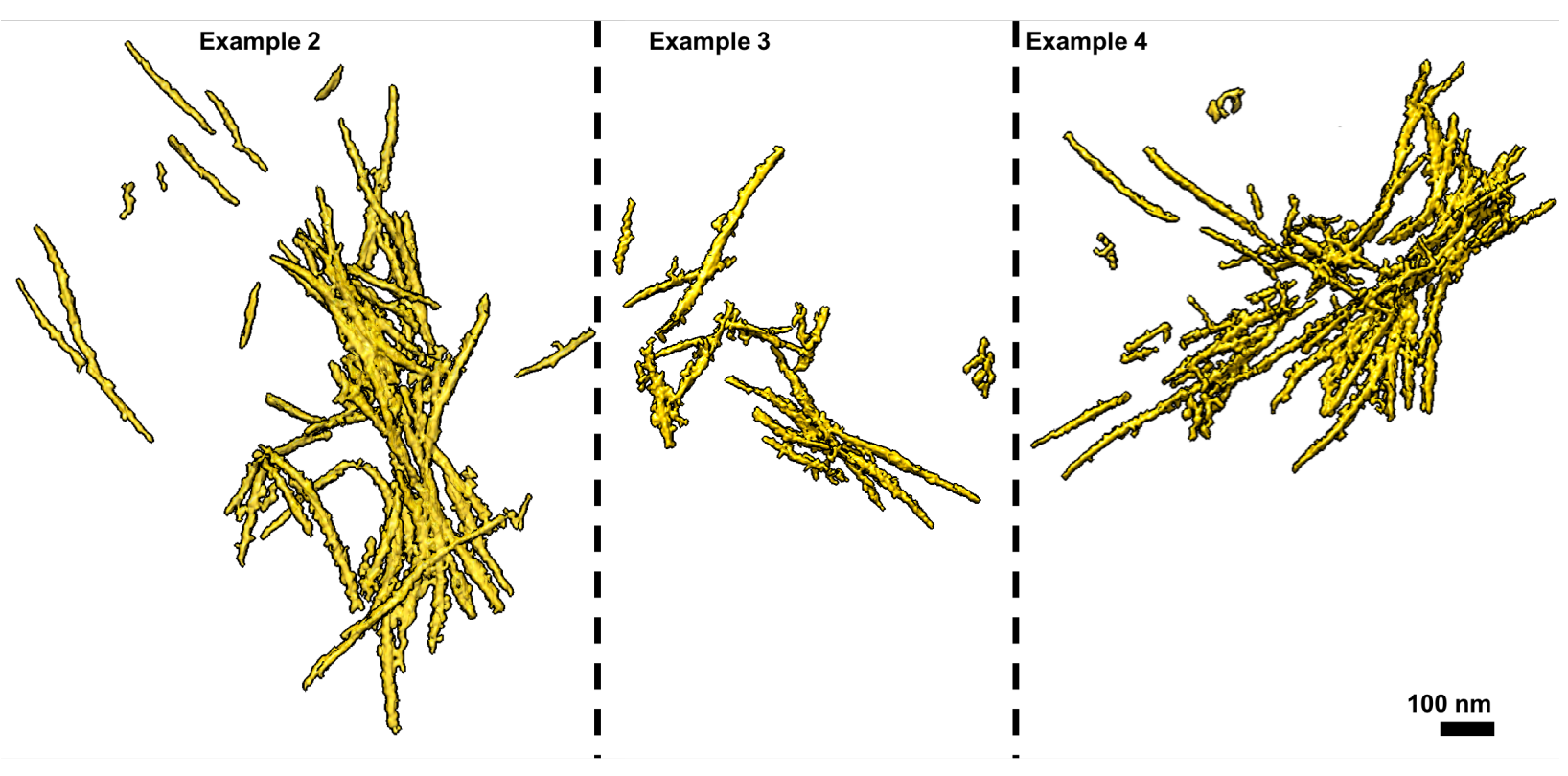

443 Supplementary Figure S2. Additional examples of mEx1-Q51 filamentous aggregates.

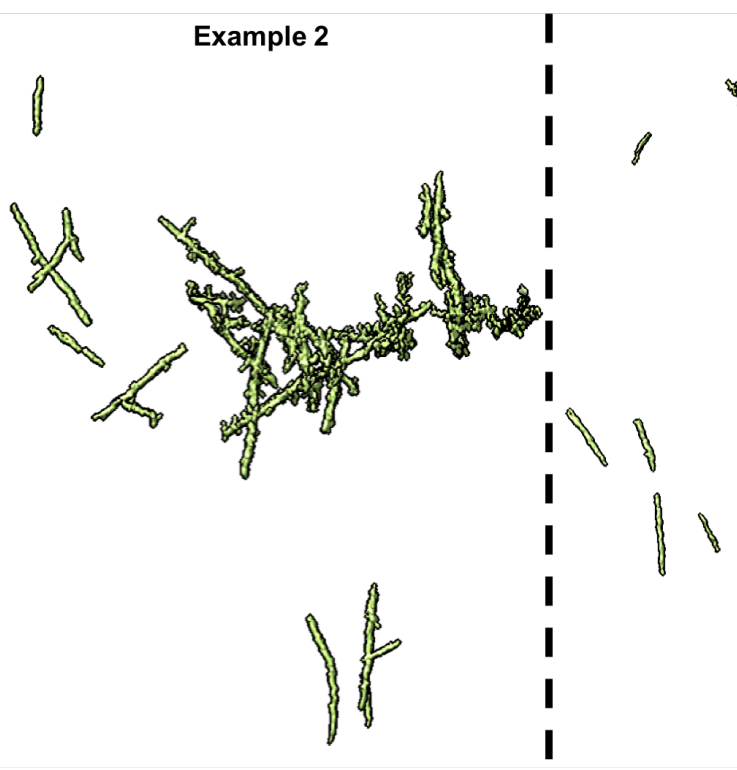

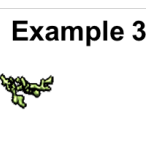

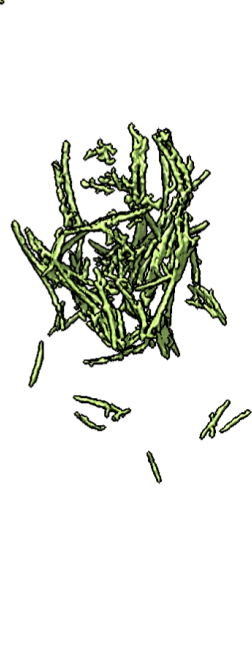

Example 4

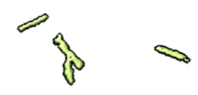

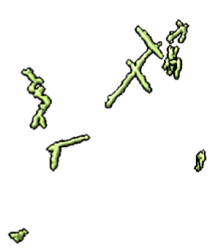

$100 \mathrm{~nm}$

446 Supplementary Figure S3. Additional examples of Q51 filamentous aggregates. 


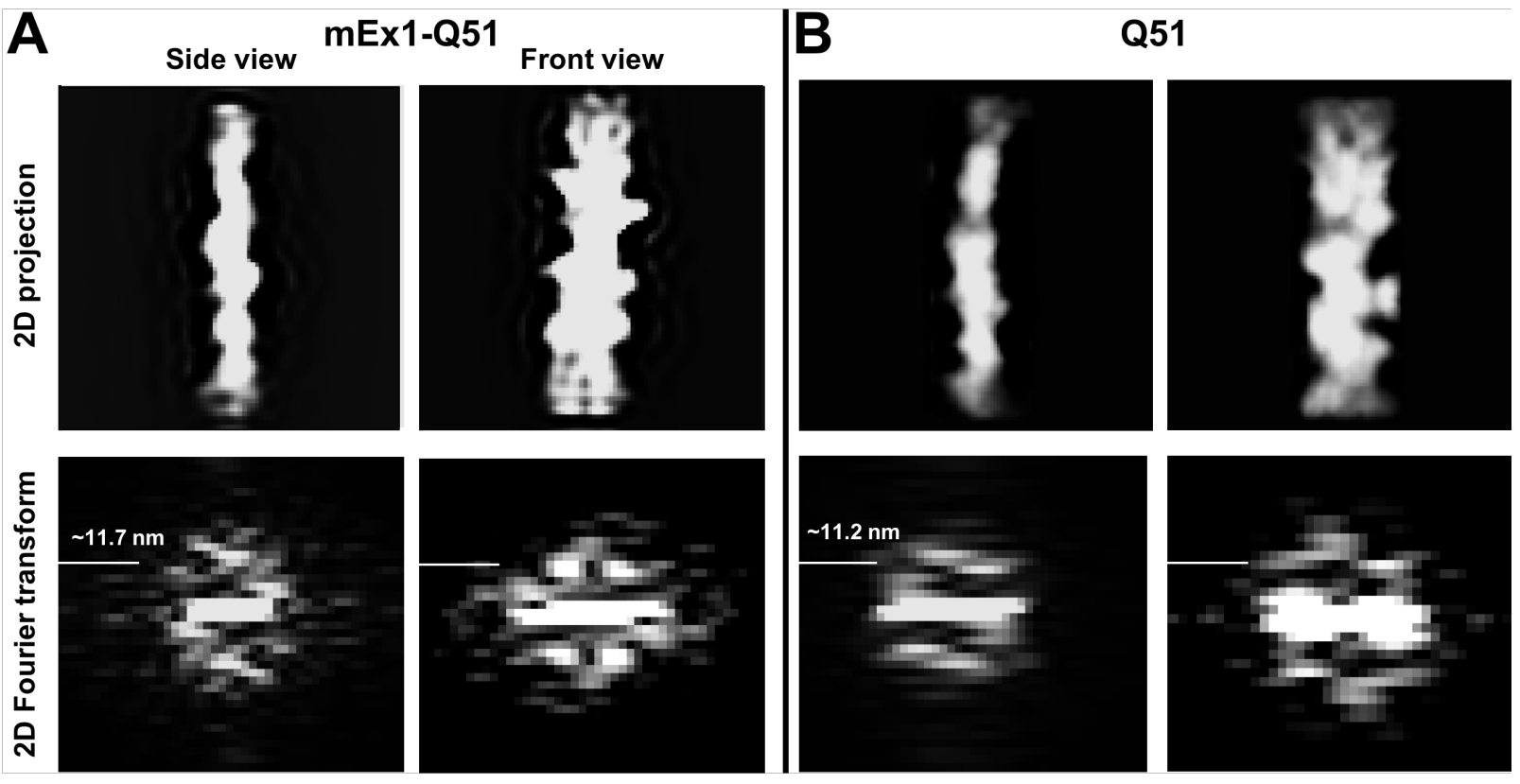

448 Supplementary Figure S4. Power spectrum of orthogonal side (left) and face-on (right)

449 projections from the subtomogram average of (A) mEx1-Q51 (Figure 2C) and (B) Q51

450 (Figure 4C) filaments. 


\section{REFERENCES}

454 Adegbuyiro, A, Sedighi, F, Pilkington, AWT, Groover, S \& Legleiter, J. (2017). Proteins Containing Expanded Polyglutamine Tracts and Neurodegenerative Disease. Biochemistry, 56(9), 1199-1217.

Albin, RL. (2017). Polyglutamine inclusion body toxicity. Mov Disord, 32(12), 1686.

Bartesaghi, A, Sprechmann, P, Liu, J, Randall, G, Sapiro, G \& Subramaniam, S. (2008). Classification and 3D averaging with missing wedge correction in biological electron tomography. Journal of Structural Biology, 162(3), 436-450.

\section{1}

462

Bäuerlein, FJB, Saha, I, Mishra, A, Kalemanov, M, Martínez-Sánchez, A, Klein, R, Dudanova, I, Hipp, MS, Hartl, FU, Baumeister, W \& Fernández-Busnadiego, R. (2017). In Situ Architecture and Cellular Interactions of PolyQ Inclusions. Cell, 171(1), 179-187.e110.

Bugg, CW, Isas, JM, Fischer, T, Patterson, PH \& Langen, R. (2012). Structural features and domain organization of huntingtin fibrils. J Biol Chem, 287(38), 31739-31746.

Chen, M, Bell, JM, Shi, X, Sun, SY, Wang, Z \& Ludtke, SJ. (2019). A complete data processing workflow for cryo-ET and subtomogram averaging. Nat Methods.

Chen, M, Dai, W, Sun, SY, Jonasch, D, He, CY, Schmid, MF, Chiu, W \& Ludtke, SJ. (2017a). Convolutional neural networks for automated annotation of cellular cryoelectron tomograms. Nat Methods, 14(10), 983-985.

Chen, S, Berthelier, V, Hamilton, JB, O'nuallain, B \& Wetzel, R. (2002). Amyloid-like features of polyglutamine aggregates and their assembly kinetics. Biochemistry, 41(23), 7391-7399. 
Chen, S, Berthelier, V, Yang, W \& Wetzel, R. (2001). Polyglutamine aggregation behavior in vitro supports a recruitment mechanism of cytotoxicity. J Mol Biol, 311(1), 173182.

Chen, Y, Wang, Z, Zhang, J, Li, L, Wan, X, Sun, F \& Zhang, F. (2017b). Accelerating electron tomography reconstruction algorithm ICON with GPU. Biophys Rep, 3(1), $36-42$.

Crick, SL, Ruff, KM, Garai, K, Frieden, C \& Pappu, RV. (2013). Unmasking the roles of $\mathrm{N}$ - and C-terminal flanking sequences from exon 1 of huntingtin as modulators of polyglutamine aggregation. Proc Natl Acad Sci U S A, 110(50), 20075-20080.

Darrow, MC, Sergeeva, OA, Isas, JM, Galaz-Montoya, JG, King, JA, Langen, R, Schmid, MF \& Chiu, W. (2015). Structural Mechanisms of Mutant Huntingtin Aggregation Suppression by the Synthetic Chaperonin-like CCT5 Complex Explained by Cryoelectron Tomography. Journal of Biological Chemistry, 290(28), 1745117461.

Davies, SW, Turmaine, M, Cozens, BA, Difiglia, M, Sharp, AH, Ross, CA, Scherzinger, E, Wanker, EE, Mangiarini, L \& Bates, GP. (1997). Formation of neuronal intranuclear inclusions underlies the neurological dysfunction in mice transgenic for the HD mutation. Cell, 90(3), 537-548.

Deng, Y, Chen, Y, Zhang, Y, Wang, S, Zhang, F \& Sun, F. (2016). ICON: 3D reconstruction with 'missing-information' restoration in biological electron tomography. Journal of Structural Biology, 195(1), 100-112. 
Difiglia, M, Sapp, E, Chase, KO, Davies, SW, Bates, GP, Vonsattel, JP \& Aronin, N. (1997). Aggregation of huntingtin in neuronal intranuclear inclusions and dystrophic neurites in brain. Science, 277(5334), 1990-1993.

Dobson, CM. (2001). The structural basis of protein folding and its links with human disease. Philos Trans R Soc Lond B Biol Sci, 356(1406), 133-145.

Duim, WC, Chen, B, Frydman, J \& Moerner, WE. (2011). Sub-diffraction imaging of huntingtin protein aggregates by fluorescence blink-microscopy and atomic force microscopy. Chemphyschem, 12(13), 2387-2390.

Duim, WC, Jiang, Y, Shen, K, Frydman, J \& Moerner, WE. (2014). Super-resolution fluorescence of huntingtin reveals growth of globular species into short fibers and coexistence of distinct aggregates. ACS Chem Biol, 9(12), 2767-2778.

Fan, H-C, Ho, L-I, Chi, C-S, Chen, S-J, Peng, G-S, Chan, T-M, Lin, S-Z \& Harn, H-J. (2014). Polyglutamine (PolyQ) diseases: genetics to treatments. Cell Transplant, 23(4-5), 441-458.

Fitzpatrick, AW \& Saibil, HR. (2019). Cryo-EM of amyloid fibrils and cellular aggregates. Curr Opin Struct Biol, 58, 34-42.

Fitzpatrick, AWP, Falcon, B, He, S, Murzin, AG, Murshudov, G, Garringer, HJ, Crowther, RA, Ghetti, B, Goedert, M \& Scheres, SHW. (2017). Cryo-EM structures of tau filaments from Alzheimer's disease. Nature, 547(7662), 185-190.

Galaz-Montoya, JG, Flanagan, J, Schmid, MF \& Ludtke, SJ. (2015). Single particle tomography in EMAN2. Journal of Structural Biology, 190(3), 279-290.

Galaz-Montoya, JG, Hecksel, CW, Baldwin, PR, Wang, E, Weaver, SC, Schmid, MF, Ludtke, SJ \& Chiu, W. (2016). Alignment algorithms and per-particle CTF 
correction for single particle cryo-electron tomography. Journal of Structural Biology, 194(3), 383-394.

521 Galaz-Montoya, JG \& Ludtke, SJ. (2017). The advent of structural biology in situby single particle cryo-electron tomography. Biophys Rep, 3(1), 17-35.

Gatchel, JR \& Zoghbi, HY. (2005). Diseases of unstable repeat expansion: mechanisms and common principles. Nat Rev Genet, 6(10), 743-755.

Gremer, L, Scholzel, D, Schenk, C, Reinartz, E, Labahn, J, Ravelli, RBG, Tusche, M, structure of amyloid-beta(1-42) by cryo-electron microscopy. Science, 358(6359), 116-119.

Gruber, A, Hornburg, D, Antonin, M, Krahmer, N, Collado, J, Schaffer, M, Zubaite, G, Lüchtenborg, C, Sachsenheimer, T, Brügger, B, Mann, M, Baumeister, W, Hartl, FU, Hipp, MS \& Fernández-Busnadiego, R. (2018). Molecular and structural architecture of polyQ aggregates in yeast. Proc Natl Acad Sci U S A, 115(15), E3446-E3453.

Hecksel, CW, Darrow, MC, Dai, W, Galaz-Montoya, JG, Chin, JA, Mitchell, PG, Chen, S, Jakana, J, Schmid, MF \& Chiu, W. (2016). Quantifying Variability of Manual Annotation in Cryo-Electron Tomograms. Microscopy and microanalysis: the official journal of Microscopy Society of America, Microbeam Analysis Society, Microscopical Society of Canada, 1-10. 
562 Jin, J, Galaz-Montoya, JG, Sherman, MB, Sun, SY, Goldsmith, CS, O'toole, ET,

Hegde, RN, Chiki, A, Petricca, L, Martufi, P, Arbez, N, Mouchiroud, L, Auwerx, J, Landles, C, Bates, GP, Singh-Bains, MK, Dragunow, M, Curtis, MA, Faull, RL, Ross, CA, Caricasole, A \& Lashuel, HA. (2020). TBK1 phosphorylates mutant Huntingtin and suppresses its aggregation and toxicity in Huntington's disease models. EMBO J, 39(17), e104671.

Hoop, CL, Lin, H-K, Kar, K, Hou, Z, Poirier, MA, Wetzel, R \& Van Der Wel, PCA. (2014). Polyglutamine amyloid core boundaries and flanking domain dynamics in huntingtin fragment fibrils determined by solid-state nuclear magnetic resonance. Biochemistry, 53(42), 6653-6666.

Hoop, CL, Lin, H-K, Kar, K, Magyarfalvi, G, Lamley, JM, Boatz, JC, Mandal, A, Lewandowski, JR, Wetzel, R \& Van Der Wel, PCA. (2016). Huntingtin exon 1 fibrils feature an interdigitated $\beta$-hairpin-based polyglutamine core. Proc Natl Acad Sci U S A, 113(6), 1546-1551.

ladanza, MG, Jackson, MP, Hewitt, EW, Ranson, NA \& Radford, SE. (2018). A new era for understanding amyloid structures and disease. Nat Rev Mol Cell Biol, 19(12), 755-773.

Iwasaki, K, Mitsuoka, K, Fujiyoshi, Y, Fujisawa, Y, Kikuchi, M, Sekiguchi, K \& Yamada, T. (2005). Electron tomography reveals diverse conformations of integrin allb $\beta 3$ in the active state. Journal of Structural Biology, 150(3), 259-267.

Jensen, EC. (2012). Use of fluorescent probes: their effect on cell biology and limitations. Anat Rec (Hoboken), 295(12), 2031-2036.

Ackerman, L, Carlson, L-A, Weaver, SC, Chiu, W \& Simmons, G. (2018). 
Neutralizing Antibodies Inhibit Chikungunya Virus Budding at the Plasma Membrane. Cell Host Microbe, 24(3), 417-428.e415.

Kremer, JR, Mastronarde, DN \& Mcintosh, JR. (1996). Computer visualization of threedimensional image data using IMOD. Journal of Structural Biology, 116(1), 71-76.

Li, B, Ge, P, Murray, KA, Sheth, P, Zhang, M, Nair, G, Sawaya, MR, Shin, WS, Boyer, DR, Ye, S, Eisenberg, DS, Zhou, ZH \& Jiang, L. (2018a). Cryo-EM of full-length alpha-synuclein reveals fibril polymorphs with a common structural kernel. Nat Commun, 9(1), 3609.

Li, Y, Zhao, C, Luo, F, Liu, Z, Gui, X, Luo, Z, Zhang, X, Li, D, Liu, C \& Li, X. (2018b). Amyloid fibril structure of alpha-synuclein determined by cryo-electron microscopy. Cell Res, 28(9), 897-903.

Lin, H-K, Boatz, JC, Krabbendam, IE, Kodali, R, Hou, Z, Wetzel, R, Dolga, AM, Poirier, MA \& Van Der Wel, PCA. (2017). Fibril polymorphism affects immobilized nonamyloid flanking domains of huntingtin exon1 rather than its polyglutamine core. Nat Commun, 8, 15462.

Lu, K, Jacob, J, Thiyagarajan, P, Conticello, VP \& Lynn, DG. (2003). Exploiting amyloid fibril lamination for nanotube self-assembly. J Am Chem Soc, 125(21), 6391-6393.

Macdonald, ME, Ambrose, CM, Duyao, MP, Myers, RH, Lin, C, Srinidhi, L, Barnes, G, Taylor, SA, James, M \& Groot, N. (1993). A novel gene containing a trinucleotide repeat that is expanded and unstable on Huntington's disease chromosomes. Cell, 72(6), 971-983. 
Maiuri, T, Woloshansky, T, Xia, J \& Truant, R. (2013). The huntingtin N17 domain is a multifunctional CRM1 and Ran-dependent nuclear and cilial export signal. Hum Mol Genet, 22(7), 1383-1394.

Mangiarini, L, Sathasivam, K, Seller, M, Cozens, B, Harper, A, Hetherington, C, Lawton, M, Trottier, Y, Lehrach, H, Davies, SW \& Bates, GP. (1996). Exon 1 of the HD gene with an expanded CAG repeat is sufficient to cause a progressive neurological phenotype in transgenic mice. Cell, 87(3), 493-506.

Mastronarde, DN. (2005). Automated electron microscope tomography using robust prediction of specimen movements. Journal of Structural Biology, 152(1), 36-51.

Nekooki-Machida, Y, Kurosawa, M, Nukina, N, Ito, K, Oda, T \& Tanaka, M. (2009). Distinct conformations of in vitro and in vivo amyloids of huntingtin-exon1 show different cytotoxicity. Proceedings of the National Academy of Sciences, 106(24), 9679-9684.

Nucifora, FC, Sasaki, M, Peters, MF, Huang, H, Cooper, JK, Yamada, M, Takahashi, H, Tsuji, S, Troncoso, J, Dawson, VL, Dawson, TM \& Ross, CA. (2001). Interference by huntingtin and atrophin-1 with cbp-mediated transcription leading to cellular toxicity. Science, 291(5512), 2423-2428.

Poirier, MA, Li, H, Macosko, J, Cai, S, Amzel, M \& Ross, CA. (2002). Huntingtin spheroids and protofibrils as precursors in polyglutamine fibrilization. J Biol Chem, 277(43), 41032-41037.

Radermacher, M. (1988). Three-Dimensional reconstruction of single particles from random and nonrandom tilt series. Journal of Electron Microscopy Technique, 9(4), 359-394. 
608 Redfern, OC, Dessailly, B \& Orengo, CA. (2008). Exploring the structure and function paradigm. Current Opinion in Structural Biology, 18(3), 394-402.

610 Rigort, A, Gunther, D, Hegerl, R, Baum, D, Weber, B, Prohaska, S, Medalia, O, Baumeister, W \& Hege, HC. (2012). Automated segmentation of electron tomograms for a quantitative description of actin filament networks. J Struct Biol, $177(1), 135-144$.

Ruggeri, FS, Vieweg, S, Cendrowska, U, Longo, G, Chiki, A, Lashuel, HA \& Dietler, G. (2016). Nanoscale studies link amyloid maturity with polyglutamine diseases onset. Sci Rep, 6, 31155.

Rusu, M, Starosolski, Z, Wahle, M, Rigort, A \& Wriggers, W. (2012). Automated tracing of filaments in 3D electron tomography reconstructions using Sculptor and Situs. J Struct Biol, 178(2), 121-128.

Sathasivam, K, Lane, A, Legleiter, J, Warley, A, Woodman, B, Finkbeiner, S, Paganetti, P, Muchowski, PJ, Wilson, S \& Bates, GP. (2010). Identical oligomeric and fibrillar

626 Scherzinger, E, Lurz, R, Turmaine, M, Mangiarini, L, Hollenbach, B, Hasenbank, R, structures captured from the brains of $\mathrm{R} 6 / 2$ and knock-in mouse models of Huntington's disease. Hum Mol Genet, 19(1), 65-78.

Scheres, SH, Zhang, W, Falcon, B \& Goedert, M. (2020). Cryo-EM structures of tau filaments. Curr Opin Struct Biol, 64, 17-25. polyglutamine expansions form amyloid-like protein aggregates in vitro and in vivo. Cell, 90(3), 549-558. 
630 Scherzinger, E, Sittler, A, Schweiger, K, Heiser, V, Lurz, R, Hasenbank, R, Bates, GP, huntingtin fragments into amyloid-like fibrils: implications for Huntingtons disease pathology. Proceedings of the National Academy of Sciences, 96(8), 4604-4609.

Schneider, R, Schumacher, MC, Mueller, H, Nand, D, Klaukien, V, Heise, H, Riedel, D, Wolf, G, Behrmann, E, Raunser, S, Seidel, R, Engelhard, M \& Baldus, M. (2011). Structural characterization of polyglutamine fibrils by solid-state NMR spectroscopy. J Mol Biol, 412(1), 121-136.

Shahmoradian, SH, Galaz-Montoya, JG, Schmid, MF, Cong, Y, Ma, B, Spiess, C,

Shen, K, Calamini, B, Fauerbach, JA, Ma, B, Shahmoradian, SH, Serrano Lachapel, IL, Chiu, W, Lo, DC \& Frydman, J. (2016). Control of the structural landscape and neuronal proteotoxicity of mutant Huntingtin by domains flanking the polyQ tract. Elife, 5.

Sivanandam, VN, Jayaraman, M, Hoop, CL, Kodali, R, Wetzel, R \& Van Der Wel, PCA. (2011). The aggregation-enhancing huntingtin N-terminus is helical in amyloid fibrils. J Am Chem Soc, 133(12), 4558-4566. Y, Wang, C-H, Chen, W, Chern, Y \& Jen-Tse Huang, J. (2015). Conformational switch of polyglutamine-expanded huntingtin into benign aggregates leads to neuroprotective effect. Sci Rep, 5, 14992. 
652 Tabrizi, SJ, Ghosh, R \& Leavitt, BR. (2019). Huntingtin Lowering Strategies for Disease Modification in Huntington's Disease. Neuron, 101(5), 801-819.

654 Testa, CM \& Jankovic, J. (2019). Huntington disease: A quarter century of progress since 655 the gene discovery. J Neurol Sci, 396, 52-68.

Vieweg, S, Ansaloni, A, Wang, ZM, Warner, JB \& Lashuel, HA. (2016). An Intein-based Strategy for the Production of Tag-free Huntingtin Exon 1 Proteins Enables New

Von Hörsten, S, Schmitt, I, Nguyen, HP, Holzmann, C, Schmidt, T, Walther, T, Bader, M, Pabst, R, Kobbe, P, Krotova, J, Stiller, D, Kask, A, Vaarmann, A, Rathke-Hartlieb, by ubiquilin. Hum Mol Genet, 15(6), 1025-1041.

Wang, L \& Sigworth, FJ. (2006). Cryo-EM and single particles. Physiology, 21(1), 13-18. 
674 Warner, JB, Ruff, KM, Tan, PS, Lemke, EA, Pappu, RV \& Lashuel, HA. (2017).

675

676

677

678

679

680

681

682

683

684

685

686

687

688

689

690

691

692

693

694

695

696

Monomeric Huntingtin Exon 1 Has Similar Overall Structural Features for WildType and Pathological Polyglutamine Lengths. J Am Chem Soc, 139(41), 1445614469.

Wetzel, R. (2012). Physical chemistry of polyglutamine: intriguing tales of a monotonous sequence. J Mol Biol, 421(4-5), 466-490.

Wild, EJ \& Tabrizi, SJ. (2017). Therapies targeting DNA and RNA in Huntington's disease. Lancet Neurol, 16(10), 837-847.

Yang, S-H, Cheng, P-H, Banta, H, Piotrowska-Nitsche, K, Yang, J-J, Cheng, ECH, Snyder, B, Larkin, K, Liu, J, Orkin, J, Fang, Z-H, Smith, Y, Bachevalier, J, Zola, SM, Li, S-H, Li, X-J \& Chan, AWS. (2008). Towards a transgenic model of Huntington's disease in a non-human primate. Nature, 453(7197), 921-924.

Yang, W, Dunlap, JR, Andrews, RB \& Wetzel, R. (2002). Aggregated polyglutamine peptides delivered to nuclei are toxic to mammalian cells. Hum Mol Genet, 11(23), 2905-2917.

Yu, Z \& Frangakis, AS. (2014). M-free: scoring the reference bias in sub-tomogram averaging and template matching. J Struct Biol, 187(1), 10-19.

Zhang, L \& Ren, G. (2012). IPET and FETR: experimental approach for studying molecular structure dynamics by cryo-electron tomography of a single-molecule structure. PloS one, 7(1), e30249.

Zhang, P. (2019). Advances in cryo-electron tomography and subtomogram averaging and classification. Curr Opin Struct Biol, 58, 249-258. 